Mufson, M. A., Manko, M. A., Kingston, J. R., and Chanock, R. M. (1961). Eaton agent pneumonia-clinical features. Fournal of the American Medical Association, 178, 369.

Vernier, R. L., Worthen, H. G., Peterson, R. D., Colle, E., and Good, R. A. (1961). Anaphylactoid purpura. I. Pathology of the skin and kidney and frequency of streptococcal infection. Pediatrics, 27, 181.

*Correspondence to Dr. S. W. Liew, Department of Paediatrics, The John Radcliffe Hospital, Oxford.

\section{The 'grey toddler': chloramphenicol toxicity}

Sir,

I read the article by Craft et al. (Archives, 1974, 49, 235) on the 'grey toddler' with interest. However, there are certain points worthy of comment.

Chloramphenicol, when given orally, provides effective therapeutic antimicrobial blood levels with good penetration in the CSF (Weinstein, 1970) and does not necessitate the intravenous route which may increase the risk of toxicity. Sulphonamides and chloramphenicol to some extent share a common metabolic pathway of glucuronide conjugation in the human liver (Seneca, 1971). This pathway is likely to be overburdened with large dosages of chloramphenicol and sulphonamides when given intravenously, which may be a contributory factor to chloramphenicol toxicity.

Two children who neither had bacterial isolation from the CSF, nor had any other supportive evidence of bacterial meningitis were continued on large doses of these drugs. The third child with Haemophilus influenzae meningitis was treated with a combination of sulphadimidine, streptomycin, and chloramphenicol; in fact chloramphenicol alone would have been sufficient for an effective therapy for this type of meningitis (Smith, 1972).

S. HAIDER

Department of Infectious Diseases, Coppetts Wood Hospital, Coppetts Wood, Muswell Hill, London N10 1JN.

\section{REFERENCES}

Seneca, H. (1971). Biological Basis of Chemotherapy of Infections and Infestations, p. 718. Davies, Philadelphia.

Smith, H. (1972). Antibiotics in Clinical Practice, 2nd ed., p. 78. Pitman, London.

Weinstein, L. (1970). Miscellaneous antimicrobial, antifungal, and antiviral agents. In The Pharmacological Basis of Therapeutics, 4th ed., p. 1270. Ed. by L. S. Goodman and A. Gilman. Macmillan, London.

Dr. Craft replies as follows:

On behalf of my co-authors I would like to reply to Dr. Haider's letter regarding our article on the 'grey toddler'. Our aim in reporting these 3 cases was to suggest that chloramphenicol may occasionally cause toxicity in toddlers, as well as in neonates, when given intravenously in a dose of about $100 \mathrm{mg} / \mathrm{kg}$ per day. We deliberately avoided the wider issue of the optimum management of a sick child with presumptive meningitis of unknown aetiology. However, we would defend the use of intravenous therapy in the initial management of any child thought to have meningitis whatever the antibiotic regimen chosen.

Chloramphenicol is excreted after glucuronation and sulphonamides following acetylation and we know of no evidence that the one interferes with the other.

A. W. CRAFT

Hospital for Sick Children, Great North Road,

Newcastle upon Tyne NE2 $3 A X$.

\section{Storage of sulphatides in gastric mucosa in the late infantile form of sulphatidosis}

Sir,

The extraneural storage of sulphatides in sulphatidosis is well known. Its presence has been reported repeatedly in the liver, gall bladder, kidneys, pancreas, adrenals (Wolfe and Pietra, 1964), and sometimes in other sites, such as the hypophysis and reticuloendothelial system. In a histochemical study of the distribution of the storage in a case of late infantile form of this disease, in a male aged 3 years, the storage of sulphatides was found in the chief cells of gastric glands predominantly in their basal portions, in addition to the above mentioned tissues. The lipid was present in a moderate amount in a form of minute, birefringent, and easily extractable droplets, staining typically brown in the Hirsch-Peiffer method. Occasionally it could be detected even in the glandular lumen. It was also present in the interstitial histiocytes, together with lipopigment. The finding of sulphatide storage in the gastric mucosa increases the list of sites of extraneural storage, and confirms the generalized nature of the metabolic defect (Hagberg and Svennerholm, 1960). If confirmed in other cases, the finding would be useful in the diagnosis of the condition during life.

M. ELLEDER
1st Department of Pathology,
Charles IV University,
2039 Albertov, Prague 2,
Czechoslovakia.

REFERENCES

Hagberg, B., and Svennerholm, L. (1960). Metachromatic leukodystrophy - a generalized lipidosis. Determination of sulphatides in urine, blood plasma and cerebrospinal fluid. Acta Paediatrica, 49, 690.

Wolfe, H. J., and Pietra, G. G. (1964). The visceral lesions of metachromatic leukodystrophy. American fournal of Pathology, 44, 921. 